\title{
Near-integrability as a numerical tool in solar system dynamics
}

\author{
M. Kaasalainen and T. Laakso \\ Observatory, PO Box 14, 00014 University of Helsinki, Finland \\ Received 4 February 2000 / Accepted 11 December 2000

\begin{abstract}
We present a simple choice of integration variables that can be used to exploit the near-integrable character of problems in celestial mechanics. The approach is based on the well-known principle of variation of parameters: instead of orbital elements, we use the phase-space coordinates the object would have at a given point in its (Keplerian) orbit if the perturbing forces were removed. This formulation is suitable for almost any numerical integrator; thus, multistep schemes are easy to build, stepsize can be adjusted, and dissipative forces are allowed. Compared with traditional non-symplectic $N$-body integrators, the approach often offers increase in speed or accuracy if perturbations are small.
\end{abstract}

Key words. methods: numerical - celestial mechanics - solar system: general

\section{Introduction}

Objects in sparsely populated systems dominated by a single massive body spend most of their time in the perturbed two-body state. Since Euler and Lagrange, dynamicists have constructed integration methods that can explicitly take into account this near-integrable character of problems in celestial mechanics. In principle, any scheme that returns the exact Kepler orbit of a two-body problem when perturbations are removed describes the system much better than a "blind" conventional $N$-body method (such as one of Gauss-Jackson or other multistep and double-integration type), the foremost advantage being a longer timestep.

No single integration method is automatically superior to others, owing to the fact that different problems usually require somewhat different approaches. However, traditional schemes modelled in cumbersome forms and variables have lately been replaced by symplectic integrators (SIs): in addition to allowing the representation of near-integrability, they exhibit no secular growth of energy error. Development in this field has been rapid in recent years, and some of the disadvantages of early SIs have been alleviated by, e.g., symplectic correctors, (limited) adjustability of stepsize, and the possibility to accommodate weak dissipative forces (see, e.g., Levison \& Duncan 1994; Saha \& Tremaine 1994; Wisdom et al. 1996; Mikkola 1997, 1998). Symplectic integrators that allow close encounters have also been constructed (Duncan et al. 1998; Chambers 1999; Mikkola \& Tanikawa 1999;

Send offprint requests to: M. Kaasalainen, e-mail: Mikko.Kaasalainen@astro.helsinki.fi
Preto \& Tremaine 1999; Levison \& Duncan 2000). However, SIs cannot by definition tackle general nonHamiltonian forces, and there is as yet no proper way of using multistep information to build inexpensive highorder schemes.

The principal manifestation of traditional methods is analytical perturbation theory (often referred to as "general perturbations"). In the numerical domain ("special perturbations") most of the traditional schemes have now little more than historical interest: they were developed for pen and paper, not for modern computing machines. However, the method known as "variation of parameters" or "variation of arbitrary constants", used in different forms by Euler, Lagrange, Poisson and many others after them (see, e.g., Herrick 1972; Danby 1987), is quite useful in its basic principle. The main question is the choice of parameters, which we discuss in this paper. The geometric Keplerian elements (and their variants) have usually been the first choice for variational formulation; however, they are not the best option for modern purposes.

We describe an approach that uses the phase-space coordinates the object would have at a given point in its (Keplerian) orbit if the perturbing forces were removed. This results in a simple near-integrable formulation that is suitable for almost any numerical integrator; one is thus free to build multistep or hybrid schemes, vary the stepsize, and add dissipative forces. This scheme is, in a way, complementary to SIs, offering an increase in speed or accuracy in problems of celestial mechanics where SIs cannot be employed.

The basic principles and concepts are presented in Sect. 2. In Sect. 3 we describe a choice of frame in 
which low-order methods are especially simple to integrate. In Sect. 4 we define another frame; in this case, any high-order multistep scheme can be efficiently applied. In Sect. 5 we discuss numerical results, and Sect. 6 sums up.

\section{Perturbative formulation}

Our goal is to seek elements $c$ such that their time derivatives $\dot{\boldsymbol{c}}$ vanish in the (collection of) two-body Sun-object system(s). For any function $\boldsymbol{c}=\boldsymbol{c}(\boldsymbol{r}, \dot{\boldsymbol{r}}, t)$, where $\boldsymbol{r}$ is the position vector and $t$ is the time, we have

$\dot{c}=\frac{\partial c}{\partial r} \dot{r}+\frac{\partial c}{\partial \dot{r}} \ddot{r}+\frac{\partial c}{\partial t}$.

But since this must identically vanish in the Keplerian case, we are left with

$\dot{\boldsymbol{c}}=\frac{\partial \boldsymbol{c}}{\partial \dot{\boldsymbol{r}}} \tilde{\ddot{\boldsymbol{r}}}$,

where $\tilde{\ddot{r}}$ denotes the part of acceleration due to perturbative forces. In heliocentric coordinates,

$\tilde{\ddot{\boldsymbol{r}}}_{i}=\sum_{k=1(i \neq k)}^{N} G m_{k}\left(\frac{\boldsymbol{r}_{i k}}{r_{i k}^{3}}-\frac{\boldsymbol{r}_{k}}{r_{k}^{3}}\right)$,

where $\tilde{\ddot{\boldsymbol{r}}}_{i}$ is the perturbative acceleration of object $i, N$ the number of objects, $G$ the gravitational constant, $m_{k}$ are the objects' masses, $\boldsymbol{r}_{i k} \equiv \boldsymbol{r}_{k}-\boldsymbol{r}_{i}$, and $\boldsymbol{r}_{k}$ are the heliocentric position vectors.

Obviously we want to choose the elements $c$ such that the partial derivatives $\partial \boldsymbol{c} / \partial \dot{\boldsymbol{r}}$ can be easily computed; also, the transformation between $\boldsymbol{c}$ and $(\boldsymbol{r}, \dot{\boldsymbol{r}})$ should be as simple as possible so that $\dot{\boldsymbol{c}}$ can be viewed in the form

$\dot{\boldsymbol{c}}=F[\boldsymbol{r}(\boldsymbol{c}, t), \dot{\boldsymbol{r}}(\boldsymbol{c}, t)]=F(\boldsymbol{c}, t)$,

i.e., in the standard form suitable for a multitude of integration schemes (note that we formulate the problem for single-integration methods instead of double-integration ones). The usual Keplerian orbital elements will not do as they are extremely cumbersome and, worst of all, lead to formulas that have singularities when eccentricity or inclination goes to zero. A practical choice is simply to derive from $(\boldsymbol{r}, \dot{\boldsymbol{r}})$ the Cartesian velocity and position the object would have at a given point in its Keplerian orbit if the perturbing forces were removed, and use them as the elements. This practicality is caused by the fact that $(\boldsymbol{r}, \dot{\boldsymbol{r}})$ at any point in a Keplerian orbit can easily be derived from those at any other point with the aid of the so-called $f$ and $g$ functions by Gauss (see Sect. 4 and Eq. (31); also Danby 1987). This makes the necessary transformations simple, and all quantities are well defined at all times.

Depending on the situation and the choice of the integration method, we use either

$\boldsymbol{c}=\left(\boldsymbol{r}_{0}, \dot{\boldsymbol{r}}_{0}\right)_{K}$ at a given time $t_{0}$ (the subscript $K$ emphasizes that the values are to be evaluated along the imagined Keplerian orbit) or

$\boldsymbol{c}=\left(\boldsymbol{r}_{0}, \dot{\boldsymbol{r}}_{0}, t_{0} ; y_{0}=0, x_{0}>0\right)_{K}$,

i.e., the position and velocity as well as the time at the point where the object would last have crossed (or would next cross) the $x z$-half plane if it moved in an unperturbed Kepler-orbit. In both cases we have six elements that can be used just like the traditional osculating geometric ones. Provided that the eccentricity is never small, one can also use the more traditional

$\boldsymbol{c}=\left(\boldsymbol{r}_{0}, \dot{\boldsymbol{r}}_{0}, t_{0} ; \dot{r}_{0}=0, \ddot{r}_{0}>0\right)_{K}$,

where $\left(\boldsymbol{r}_{0}, \dot{\boldsymbol{r}}_{0}\right)$ can be given in spherical coordinates, and $t_{0}$ is now the time of perihelion. This is useful for highly eccentric osculating ellipses that can occasionally open into hyperbolae.

\subsection{Variable time step}

A basic method of controlling the length of the time step is to use the standard procedure of extending phase space by introducing a new independent variable $\tau$, related to the time $t$ by the differential equation

$\frac{\mathrm{d} t}{\mathrm{~d} \tau}=g(\boldsymbol{c}, t)$,

where $g$ is any given function (e.g., proportional to velocity) that can depend explicitly on time as well. The differential equations of Eq. (4) are now replaced by

$\frac{\mathrm{d} \boldsymbol{c}}{\mathrm{d} \tau}=g(\boldsymbol{c}, t) F(\boldsymbol{c}, t)$,

while the time corresponding to $\tau$ is obtained by integrating (8). The stepsize for $\tau$ can be constant.

\subsection{Variational equations}

To obtain either the first Liapunov exponent or the state transition matrix (called matrizant in Danby 1987; also see, e.g., Mikkola \& Innanen 1999), one needs to compute the evolution of the differences $\boldsymbol{d}_{\boldsymbol{c}}$ between the values of $\boldsymbol{c}$ for two initially close orbits. This is governed by the variational equations

$\dot{d}_{c}=M d_{c}$

where the matrix $M=\partial \dot{\boldsymbol{c}} / \partial \boldsymbol{c}$ (see, e.g., Lichtenberg \& Lieberman 1991). In our case it assumes the form

$M_{i j}=\frac{\partial^{2} c_{i}}{\partial \boldsymbol{w} \partial \dot{\boldsymbol{r}}} \frac{\partial \boldsymbol{w}}{\partial c_{j}}+\frac{\partial c_{i}}{\partial \dot{\boldsymbol{r}}} \frac{\partial \tilde{\ddot{r}}}{\partial \boldsymbol{r}} \frac{\partial \boldsymbol{r}}{\partial c_{j}}$,

where $\boldsymbol{w} \equiv(\boldsymbol{r}, \dot{\boldsymbol{r}})$ (the compact vector notation in (11) is somewhat unorthodox but rather obvious). The differences $\boldsymbol{d}_{\boldsymbol{c}}$ can be computed along with $\boldsymbol{c}$ using the same integration method. By definition, the differential Eq. (10) 
describes infinitesimal quantities: its linearity makes $\boldsymbol{d}_{\boldsymbol{c}}$ scale-free. The first Liapunov exponent $\sigma$ is formally defined as

$\sigma=\lim _{t \rightarrow \infty} \frac{1}{t} \ln \frac{\left|\boldsymbol{d}_{\boldsymbol{c}}\right|}{\left|\boldsymbol{d}_{\boldsymbol{c}}(0)\right|}$

Rather than integrating the full variational equations, the evolution of the variations can also be computed by differentiating the orbit integration algorithm directly (Mikkola \& Innanen 1999).

It is perhaps worth noting that the Liapunov exponent is usually defined for autonomous systems. If there is explicit time-dependence, one should in principle perform the above trick of extending phase space. If the expansion is trivial, i.e., $g \equiv 1$ in (8) to make the system formally autonomous, we have $\dot{d}_{\mathrm{t}}=0$, so we can set $d_{\mathrm{t}}=0$ and ignore it.

\section{Fixed $t_{0}$ and low-order methods}

The partial derivatives $\partial \boldsymbol{c} / \partial \dot{\boldsymbol{r}}$ in (2) become trivial (either 1 or 0 ) if we choose to employ $t_{0}=t$, i.e., a point in the actual orbit is also taken to be its own reference point in the Kepler orbit. Thus

$$
\frac{\mathrm{d} \boldsymbol{r}_{0}}{\mathrm{~d} t}=0, \quad \frac{\mathrm{d} \dot{\boldsymbol{r}}_{0}}{\mathrm{~d} t}=\tilde{\ddot{\boldsymbol{r}}}
$$

(Note carefully that $\mathrm{d} \boldsymbol{r}_{0} / \mathrm{d} t \neq \dot{\boldsymbol{r}}_{0}-$ the two quantities are fundamentally different.) This minimizes the work at each point and shifts the computational load to the Keplerian transformations (via the $f, g$-functions) needed to bring other points to the same $t_{0}$-frame. Note that $\boldsymbol{r}_{0}$ only changes by such transformations and not by a differential equation. For example, a naive first-order Euler step consists of a "drift" in the Kepler part to bring the previous point to the new frame followed by a "kick" from the perturbative part.

The matrix $M$ in (11) becomes especially simple in this approach as the double derivatives vanish and the only remaining nontrivial derivatives are $\partial \tilde{\ddot{\boldsymbol{r}}} / \partial \boldsymbol{r}$. Just like $\boldsymbol{r}_{0}, \boldsymbol{d}_{\boldsymbol{r}_{0}}$ evolves only by transformation from one frame to another (i.e., not by integration). Since $\boldsymbol{d}_{\boldsymbol{c}}$ represent infinitesimal quantities (differentials), the transformation is given by

$\overline{\boldsymbol{d}}_{\boldsymbol{r}_{0}}=\frac{\partial \overline{\boldsymbol{r}}_{0}}{\partial \boldsymbol{c}} \boldsymbol{d}_{\boldsymbol{c}}$

where the bar over a quantity denotes its value in the frame associated with the new point; the derivatives are readily obtained from the $f, g$ functions (see Sect. 4). This transformation thus retains the scale-free linearity of $\boldsymbol{d}_{\boldsymbol{c}}$ in the differential equation.

For higher orders one usually employs multistep schemes to keep the force evaluations at minimum; in any case, about $n$ points at different epochs are typically needed to construct an $n$ th-order integrator. One would thus require about $n$ transformations per step in the $t_{0}=t$-approach, so the computational overhead from the $f, g$-calculations quickly neutralizes the gain from the simple derivatives as the order of the method increases. Therefore the $t_{0}=t$-frame is suitable only for low-order methods such as the modified midpoint method in a Bulirsch-Stoer integrator (Press et al. 1989). Since the errors in integration - especially the energy error in conservative systems - are best kept small by using a high-order integrator, long integrations are not very suitable for the $t_{0}=t$-frame. Thus the simple formulation for the variational equations is mainly applicable to the state transition matrix (for obtaining nearby trajectories) during relatively short integration times.

\section{Fixed $y_{0}$ and higher-order methods}

A common reference frame for all points gets rid of the excessive $f, g$-transformations. Setting $t_{0}=0$ for all points is not a practical way to establish such a frame unless the total integration time is very short. This is because errors will be measured against just one orbital cycle rather than the whole time span as Kepler's equation is solved: what is small relative to, say, $2000 \pi$ is not insignificant relative to $2 \pi$. Since we would have to perform coordinate transformations over many orbital cycles, the slightest errors would be exponentially amplified and finally blow up. Using a new $t_{0}$ every now and then to prevent transformations over long time spans would not be very practical either, for then the points would be only "piecewise" in a common frame of reference, and one would have to do extra transformations near the interfaces of the pieces to establish continuity.

If we choose $\boldsymbol{c}=\left(\boldsymbol{r}_{0}, \dot{\boldsymbol{r}}_{0}, t_{0} ; y_{0}=0, x_{0}>0\right)_{K}$ as the common frame, we will have no difficulties as we will not have to do transformations over more than one cycle if we do not want to. Now $t_{0}$ 's role among the new elements resembles that of the time of perihelion among the traditional ones. Since no "perihelion" is needed now, this frame is especially suitable for orbits at low eccentricities.

An expression for $t_{0}$ can be found using the $f$ and $g$ functions. Using the subscript 0 for these functions to emphasize that we shift from $(\boldsymbol{r}, \dot{\boldsymbol{r}})$ to $\left(\boldsymbol{r}_{0}, \dot{\boldsymbol{r}}_{0}\right)$, we have

$\boldsymbol{r}_{0}=f_{0} \boldsymbol{r}+g_{0} \dot{\boldsymbol{r}}$

where

$f_{0}=1-\frac{a}{r}(1-\cos \hat{E})$,

$g_{0}=\Delta t-\frac{a^{3 / 2}}{\sqrt{\mu}}(\hat{E}-\sin \hat{E})$

and $\mu=G\left(m_{i}+m_{0}\right), a=-1 / \alpha, \alpha=|\dot{\boldsymbol{r}}|^{2} / \mu-2 / r$. Also, $\Delta t=t_{0}-t$ and $\hat{E}$ is the corresponding difference between the eccentric anomalies at $\boldsymbol{r}_{0}$ and $\boldsymbol{r}$ (note that the absolute values of the eccentric anomalies are never needed and that $\hat{E}$ is always well defined while $E$ is not). Solving for 
$\Delta t$ in the difference-formed Kepler's equation (see, e.g., Danby 1987)

$\frac{\sqrt{\mu}}{a^{3 / 2}} \Delta t=\hat{E}+\frac{u}{\sqrt{\mu a}}(1-\cos \hat{E})-s \sin \hat{E}$,

where $u=\boldsymbol{r} \cdot \dot{\boldsymbol{r}}$ and $s=1+\alpha r$, we obtain

$g_{0}=\frac{u a}{\mu}(1-\cos \hat{E})+r \sqrt{\frac{a}{\mu}} \sin \hat{E}$.

Since we have set $y_{0}=0$, we know that

$f_{0} y+g_{0} \dot{y}=0$

so, using the new form (19) for $g_{0}$, we have

$y+A(1-\cos \hat{E})+B \sin \hat{E}=0$,

where

$A=\frac{u a}{\mu} \dot{y}-\frac{a}{r} y, \quad B=r \sqrt{\frac{a}{\mu}} \dot{y}$.

Solving for $\sin \hat{E}$ and $\cos \hat{E}$ (and requiring that $x_{0}>0$ when $y_{0}=0$ ), we finally obtain $\hat{E}$ from

$\sin \hat{E}=\frac{-B(A+y)+A \sqrt{B^{2}-2 A y-y^{2}}}{A^{2}+B^{2}}$

and

$\cos \hat{E}=\frac{A(A+y)+B \sqrt{B^{2}-2 A y-y^{2}}}{A^{2}+B^{2}}$.

The above formulae hold for prograde motion $\left(L_{z}>0\right)$; if the motion is retrograde, the branch sign immediately in front of the square root terms is changed to negative (note that $\hat{E}$ always has the same sign as $\Delta t$ ). $A^{2}+B^{2}$ as well as the square root are positive definite (for elliptic motion), so the formulae hold everywhere. Substituting $\hat{E}$ to (18) or to $g_{0}$ in (17), we get $\Delta t$ and thus

$t_{0}=t+\Delta t$.

From (15) we obtain $\boldsymbol{r}_{0}$, while $\dot{\boldsymbol{r}}_{0}$ is found from

$\dot{\boldsymbol{r}}_{0}=\dot{f}_{0} \boldsymbol{r}+\dot{g}_{0} \dot{\boldsymbol{r}}$

where

$\dot{f}_{0}=-\frac{\sqrt{\mu a} \sin \hat{E}}{r r_{0}}$,

$\dot{g}_{0}=1-\frac{a}{r_{0}}(1-\cos \hat{E})$.

The integration procedure is now as follows: from the initial values of $(\boldsymbol{r}, \dot{\boldsymbol{r}})$ we get the corresponding $\boldsymbol{c}=$ $\left(t_{0}, \boldsymbol{r}_{0}, \dot{\boldsymbol{r}}_{0}\right)$ to be integrated with whatever numerical method we have chosen. The derivatives $\dot{c}$ are given by (2); using (15) and (26) we can write $\dot{\boldsymbol{c}}$ as

$\frac{\mathrm{d} \hat{\boldsymbol{r}}_{0}}{\mathrm{~d} t}=\left(\frac{\partial \hat{f}_{0}}{\partial \dot{\boldsymbol{r}}} \cdot \tilde{\ddot{\boldsymbol{r}}}\right) \boldsymbol{r}+\left(\frac{\partial \hat{g}_{0}}{\partial \dot{\boldsymbol{r}}} \cdot \tilde{\ddot{\boldsymbol{r}}}\right) \dot{\boldsymbol{r}}+\hat{g}_{0} \tilde{\ddot{\boldsymbol{r}}}$ and

$\frac{\mathrm{d} t_{0}}{\mathrm{~d} t}=\frac{\partial t_{0}}{\partial \dot{\boldsymbol{r}}} \cdot \tilde{\ddot{\boldsymbol{r}}}$,

where the hat over $f_{0}, g_{0}, \boldsymbol{r}_{0}$ is either uniformly read as a dot or ignored everywhere.

The values of $\boldsymbol{r}, \dot{\boldsymbol{r}}$ needed in computing the derivatives (and thus obtained as "by-products") are given by

$\boldsymbol{r}=f \boldsymbol{r}_{0}+g \dot{\boldsymbol{r}}_{0}, \quad \dot{\boldsymbol{r}}=\dot{f} \boldsymbol{r}_{0}+\dot{g} \dot{\boldsymbol{r}}_{0}$,

where $f, g$ are defined as $f_{0}, g_{0}$ above but, of course, with $(\boldsymbol{r}, \dot{\boldsymbol{r}})$ and $\left(\boldsymbol{r}_{0}, \dot{\boldsymbol{r}}_{0}\right)$ interchanged everywhere. $\hat{E}$ is in this case obtained by solving Kepler's Eq. (18) (with $\Delta t=$ $\left.t-t_{0}\right)$. One should used the fast, quartically convergent iteration technique (Danby 1987) that will require only a couple of iterations. A suitable initial guess is, e.g., $\hat{E}_{0}=$ $\hat{E}_{\text {prev }}+\sqrt{\mu} a^{-3 / 2}\left(t-t_{\text {prev }}\right)$, "prev" referring to the previous point.

One must not let the integrated $t_{0}$ fall too much behind the actual time $t$ lest the errors accumulate; this is prevented by adding multiples of periods to an old $t_{0}$ in the same way as one would set a new perihelion time every now and then even if all the other elements remained unchanged (or by finding a "fresh" $t_{0}$ regularly during the integration from some obtained $(\boldsymbol{r}, \dot{\boldsymbol{r}})$ ). This does not disturb the calculations in any way, and the elements of all points are always in the same frame of reference.

If the osculating perihelion is always well defined, we can use the frame $(7)$ and replace $\hat{E}$ by the absolute value of eccentric anomaly so that $t_{0}$ becomes the time of perihelion. When working with highly eccentric orbits, the "universal formulation" via Stumpff functions (Danby 1987) may be easier than using trigonometric/hyperbolic functions.

\section{Numerical examples}

We discuss here simple numerical examples that illustrate the basic properties of the perturbative formulation. It is not in the scope of this short study to present a detailed analysis of some chosen system: the main emphasis is on the choice of coordinates that represent a near-integrable system, not on the technical details of the integration method used.

As an example of the $y_{0}=0$-frame, a multistep method, and a dissipative force, we consider a satellite experiencing a drag force $-k \dot{\boldsymbol{r}}$ (for orbits of low eccentricity, this frame is not just useful but necessary when multistep methods are used). With the perturbative scheme, the spiralling orbit can obviously be computed with considerably fewer steps than in the non-perturbative approach. An interesting quantity is the magnitude of this advantage as a function of the coefficient $k$. We used a basic AdamsBashforth multistep method to integrate the orbit with different values of $k$ and required tolerance. Regardless of the tolerance and the order of the method, the ratio of the stepsize in the perturbative approach to that in the 
direct one was typically an exponential function of $\log _{10} k$. For example, with a fifth-order method and an accuracy of one part in $10^{11}$, the perturbative scheme could use a 50-100 times longer step at $k=10^{-6}$ ( $k$ scaled to be roughly descriptive of the proportional strength of the perturbation), while at $k=10^{-4}$ the ratio was about 20 , and even at $k=0.01$ the steps could be some five times longer. A rough rule of thumb for the stepsize ratio $\mathcal{R}$ (at $k \leq 0.01)$ is

$\mathcal{R} \approx 10 \times 2^{-\left(3+\log _{10} k\right)}$.

Thus the benefits of the near-integrable approach are clear even when the perturbation is no longer small and the equations of motion are far from analytically integrable. Using $g$ in (8) does not really alter the ratio of the steplengths since much the same $g$ can be used for both the perturbative and the direct approach (in the above case, a practical value for $g$ is inversely proportional to the object's speed or even its angular speed).

As an example of the $t_{0}=t$-frame, we computed orbit values for asteroids in eccentric orbits using the Bulirsch-Stoer extrapolation scheme with the modified midpoint method (Press et al. 1989). As in the previous example, we used varying perturbation strengths (in some cases "Jupiter" was tens of times more massive than in reality) and tolerance levels. In this case, the near-integrable/direct stepsize ratio had no clear correlation with these (except for very small perturbations and low accuracies, of course); this was mostly due to the strongly varying strengths of perturbations as the asteroid proceeded in its orbit. We found that, on average, the timestep required for the near-integrable approach can be some five times longer than in the direct method. Similar results are obtained with spatially fixed frames and multistep methods. For orbits not close to giant planets (i.e., typically at relatively low eccentricities or high inclinations), the stepsize ratio can be about ten; the rule of thumb above seems to apply rather generally. Even though the steps take a longer time to compute in our approach due to the Keplerian transformations, the overall speed of the algorithm is usually noticeably faster than that of direct integration.

Eccentric orbits bring about the problem of close encounters. One can, in principle, switch to a new dominating central body whenever necessary and choose a suitable near-integrable Keplerian configuration. However, we have found that all of the time during a "moderately close" encounter is spent in the transition zone where no single body dominates the gravitational field strongly enough for the near-integrable approach to be efficient. For an asteroid approaching Jupiter, this zone is roughly between 0.02 AU and 1 AU from the planet. "Proper" close encounters are thus very rare, and even in them the time spent in the transition zone dominates the computational effort. A further problem is that the object may wander in and out of the transition zone several times during the encounter. Close encounters are thus clearly easiest to handle in conventional integration variables.
Whenever the integrator finds that the strength of the perturbation gets too large - typically well over a hundredth part of the dominating force - it switches to "ordinary mode". Frequent close encounters undermine the efficiency of near-integrability, so only an object not engaged in continuous interplay with Jupiter can be integrated well in this scheme.

To summarize, our numerical tests show that the nearintegrable approach is efficient whenever the perturbations do not considerably exceed about a hundredth part of the force caused by the dominating body. Occasional close encounters are no obstacle, but they must be handled with a separate method. Thus the impact of this approach should be greatest in integrating orbits (perturbed also by dissipative forces) that mostly stay away from the transition tori surrounding the orbits of the giant planets. Orbits with high eccentricities can be efficiently integrated if their inclinations are suitable.

\section{Conclusions}

The primary motivation for our study was to find out whether there is a way of formulating a near-integrable non-symplectic scheme more efficient than the direct $N$ body computation. The traditional perturbative methods are now passé, but some of their basic principles can still be used for efficient numerical computation. Since the straightforward formulation presented here is based on the choice of the integrated variables, the actual integration method can be chosen quite freely. The simplest frame is the one with $t_{0}=t$, best used with low-order extrapolation methods such as Bulirsch-Stoer; the longest integration steps are allowed by high-order multistep schemes in a spatially fixed frame.

The error in integration depends on the specific method chosen; not much can be said about the error elements introduced by the generic principle. Various numerical experiments indicate that (e.g., in the case of our solar system) the stepsize can be sizably larger in this approach than in a non-perturbative one to cause similar error magnitudes in the two methods. The main limitation is, of course, the strength of the perturbation: roughly speaking, its maximum value for efficient use is of the order of one percent of the dominating force.

Integrators based on near-integrability are somewhat more efficient than direct ones, but this advantage is not as clear as that provided by symplectic integrators especially in long-term integrations. The special characteristics exhibited by SIs in symplectic systems are, indeed, quite remarkable, and due to a "deeper" connection with the dynamics of the system than mere near-integrability and conservation of energy. However, when SIs cannot be used, the next best thing to do to maintain some knowledge about the nature of the system may often be to use the near-integrable formulation. In some cases(especially in dissipative systems) the advantage gained can be considerable. 


\section{References}

Chambers, J. E. 1999, MNRAS, 304, 793

Danby, J. M. A. 1987, Fundamentals of Celestial Mechanics (Willman-Bell, Richmond)

Duncan, M., Levison, H. F., \& Lee, M. H. 1998, AJ, 116, 2067 Herrick, S. 1972, Astrodynamics (Van Nostrand, New York) Levison, H. F., \& Duncan, M. J. 1994, Icarus, 108, 18

Levison, H. F., \& Duncan, M. J. 2000, AJ, 120, 2117

Lichtenberg, A. L., \& Lieberman, M. A. 1991, Regular and Stochastic Motion (Springer, New York)
Mikkola, S. 1997, Celest. Mech., 67, 145

Mikkola, S. 1998, Celest. Mech., 68, 249

Mikkola, S., \& Innanen, K. 1999, Celest. Mech., 74, 59

Mikkola, S., \& Tanikawa, K. 1999, Celest. Mech., 74, 287

Press, W., Flannery, B., Teukolsky, S., \& Vetterling, W. 1989, Numerical Recipes (Academic Press, New York)

Preto, M., \& Tremaine, S. 1999, AJ, 118, 2532

Saha, P., \& Tremaine, S. 1994, AJ, 108, 1962

Wisdom, J., Holman, M., \& Touma, J. 1996, Fields Instit. Commun. 10, 217 